\title{
Classification of Enterobacteriaceae by minimization of stochastic complexity
}

\author{
H. G. Gyllenberg, ${ }^{1}$ M. Gyllenberg, ${ }^{2}$ T. Koski,, ${ }^{2,3}$ T. Lund, ${ }^{2}$ J. Schindler ${ }^{4}$ \\ and M. Verlaan ${ }^{5}$
}

Author for correspondence: M. Gyllenberg. Tel: +3582333 6567. Fax: +35823336595.

e-mail:matsgyl@utu.fi

1 Institute of Biotechnology, University of Helsinki, 00014 Helsinki, Finland

2 Department of Mathematics, University of Turku, 20014 Turku, Finland

3 Department of Mathematics, Royal Institute of Technology, 10044 Stockholm, Sweden

4 Department of Medical Microbiology, 3. Medical Faculty, Charles University, Srobarova, 50, Prague 10, Czech Republic

5 Faculty of Technical Mathematics and Informatics, Delft University of Technology, 2600 GA Delft, The Netherlands

\begin{abstract}
A new method for classifying bacteria is presented and applied to a large set of biochemical data for the Enterobacteriaceae. The method minimizes the bits needed to encode the classes and the items or, equivalently, maximizes the information content of the classification. The resulting taxonomy of Enterobacteriaceae corresponds well to the general structure of earlier classifications. Minimization of stochastic complexity can be considered as a useful tool to create bacterial classifications that are optimal from the point of view of information theory.
\end{abstract}

Keywords: classification, Enterobacteriaceae, information theory, stochastic complexity, taxonomy

\section{INTRODUCTION}

According to Rissanen (1989) the best theory (or model) to explain a given set of data is the one which minimizes the sum of (1) the length in bits of the description of the theory, and (2) the length in bits of the description of the data within the theory or model. This statement can be viewed as a formalization, programmable on a computer, of Occam's razor, the principle that tells us not to introduce more concepts than necessary to explain observed facts. Classifying a collection of items according to some specified method (classification model) can be viewed as a means for encoding information about the data. Following the above-mentioned principle of Rissanen, the best classification is therefore the one which requires the least number of bits to code the classification with respect to the model chosen and to code the items within the classification. The relevant

\footnotetext{
Abbreviations: SC, stochastic complexity; CFARM, classification of Farme et al. (1985); HMO, hypothetical mean organism; ENTE, Enterobacteriaceae material; ESSY, escherichias, salmonellas, shigellas and yersinias from ENTE; COLI, E. coli strains from ENTE; SCENTE, SCESSY, SCCOLI, SC-minimizing classification of ENTE, ESSY, COLI.
}

mathematical quantity describing the minimum number of bits is that of stochastic complexity (SC) (Rissanen, 1989).

Gyllenberg et al. (1994a) (see also Gyllenberg et al., 1993 and Gyllenberg \& Koski, 1996) gave a precise description of the mathematical model corresponding to probabilistic numerical identification in phenetic bacterial taxonomy (Dybowski \& Franklin, 1968; Lapage et al., 1973; Willcox et al., 1980). The 'best' taxonomy according to Rissanen's principle is therefore the one that minimizes SC with respect to this model.

A good classification should have an information content as large as possible (Pankhurst, 1991; Sneath, 1995a, b). Gyllenberg et al. (1994b) showed that minimizing SC amounts to maximizing the information content of the classification. Thus increasing SC implies loss of information whereas decreasing SC indicates gain in information content. Hence SC provides a means to compare different classifications of a given collection of items and to select the best (from an information theory point of view) classification among different alternatives. Since the total number of possible classifications is finite, albeit extremely large, the SC has an 
absolute minimum and thus it is, at least in principle, possible to find the very best (again from the point of view of information theory) classification of the data set under consideration. We emphasize that neither is the number of classes given beforehand, nor is there any $a$ priori given level of similarity that two items have to exceed in order to be placed into the same group. The number of classes is determined solely from the requirement of minimizing the SC.

The purpose of this paper is to demonstrate the applicability of SC minimization as a classification method in microbiological taxonomy by comparing its outcome with a generally applied classification based on other principles. The family Enterobacteriaceae was chosen for demonstration for two reasons: first, because Enterobacteriaceae constitute a particularly well-studied group of bacteria the taxonomy of which can be considered to be rather stable; and second, because the abundant availability of data on Enterobacteriaceae allowed the utilization of a relevantly large set of test material.

The Enterobacteriaceae classification of Farmer et al. (1985) was chosen as the main reference. Both genotypic and phenotypic models were applied in the work of Farmer et al. (1985), e.g. probabilistic numerical identification in the spirit of Lapage et al. (1973). All species and genus names used in this paper follow the nomenclature of Farmer et al. (1985). The discussions of Enterobacteriaceae (Brenner, 1992) in Bergey's Manual (Krieg \& Holt, 1984) and The Prokaryotes (Balows et al., 1992) are in accordance with the conclusions of Farmer et al. (1985). The comparability of the results presented in this paper and those of Farmer et al. (1985) was secured by the fact that the present authors used the binary codes of isolates from the files of Farmer et al. (1985). The classification of Farmer et al. (1985) is referred to as CFARM in subsequent sections of this paper.

\section{METHODS}

Material. Altogether data of 5313 strains of Enterobacteriaceae representing 104 species or corresponding biogroups were included in the study. The source of the material (which is listed in Table 1) was the database of Enterobacteriaceae and Vibrionaceae compiled from 1972 to 1989 by the Enteric Bacteriology Laboratories, CDC, Atlanta, GA, USA. There were 67 un-named strains of Shigella in the material. We decided to include them in the runs, and in the files these strains were designated as Shigella prov(isional).

The data consisted of 47 binary characters (biochemical reactions) for each specimen (strain, isolate); the characters were the same as those described by Farmer et al. (1985) and in the binary codes they are presented in the same order as by Farmer et al. (1985). Since details of the characters are irrelevant for the purposes of the present paper, readers are referred to Farmer et al. (1985) for this information. Some data were missing in the specimen codes (total frequency: $1.55 \%$ ). Missing data occurred mainly in isolates from the early 1970 s and in character 46 (yellow pigment) for 2803 specimens. Missing bits were replaced by either 0 or 1 determined by coin tossing. It could be concluded that this did not affect the final results (when character 46 and the oldest isolates were omitted the material contained almost no missing data and the classification method gave rise to outcomes almost identical with those obtained using the complete material).

Description of classes. The centroid of a class is by definition the vector giving the frequencies of 1 s for the different attributes. Rounding off each component of the centroid to the nearest integer ( 0 or 1 ) one obtains the hypothetical mean organism (HMO) of that class (Gower, 1974; Sneath, 1979). As a measure of the heterogeneity of a class we chose its distortion, which is the mean number of bits by which the members of the class differ from the HMO.

Mathematical and computational methods. We describe mathematically a classification of strains with $d$ binary (0 or 1$)$ features into $k$ classes by the numbers

$\lambda_{1}, \lambda_{2}, \ldots, \lambda_{k}\left(\lambda_{1}+\ldots+\lambda_{k}=1\right)$

and

$\theta_{i j} ; i=1, \ldots, d ; j=1, \ldots, k,\left(0 \leqslant \theta_{i j} \leqslant 1\right)$,

where $\lambda_{j}$ is the relative frequency of strains in the $j^{\text {th }}$ class and $\theta_{i j}$ is the relative frequency of $1 \mathrm{~s}$ in the $i^{\text {th }}$ position in the $j^{\text {th }}$ class. The centroid of the $j^{\text {th }}$ class is the vector $\left(\theta_{1 j}, \theta_{2 j}, \ldots\right.$, $\left.\theta_{d j}\right)$. The distribution of feature vectors $\mathrm{x}=\left(x_{1}, x_{2}, \ldots, x_{d}\right)$, $\left(x_{i}=1\right.$ or 0$)$ of strains in class $j$ is given by

$p_{j}(\mathbf{x})=\prod_{i=1}^{d}\left(1-\theta_{i j}\right)^{1-x_{i}} \theta_{i j}^{x_{i}}$

(Dybowski \& Franklin, 1968; Wilcox et al., 1980). As a statistical model of the classification we therefore choose the distribution

$p(\mathbf{x})=\sum_{j=1}^{k} \lambda_{j} p_{j}(\mathbf{x})$

with the numbers $k, \lambda_{j}$ and $\theta_{i j}$ being the parameters of the model. We emphasize that this statistical representation is simply a mathematically convenient way of defining the classification model and it does not imply any randomness in the data.

It was shown by Gyllenberg et al. (1994b) that the stochastic complexity SC of a set of $t$ strains with respect to the above model is

$\mathrm{SC}=\log \frac{k(k+1) \cdots(t+k-1)}{t_{1} ! t_{2} ! \cdots t_{k} !}+\sum_{j=1}^{k} \sum_{i=1}^{d} \log \frac{t_{j} !}{t_{i j} !\left(t_{j}-t_{i j}\right) !}$

where $t_{j}$ is the number of strains in class $j$ and $t_{i j}$ is the number of strains in class $j$ with the $i^{\text {th }}$ feature equal to 1 (log denotes the logarithm to the base 2). The first term in equation (3) describes the complexity of the classification and the second term the complexity of the strains with respect to the classification.

Gyllenberg et al. (1994b) also showed that minimizing the SC with respect to the model (2) amounts to maximizing the information content of the classification.

Even if SC has a well-defined minimum it is practically impossible to find the classification at which it is attained, since an exhaustive enumeration of all classifications is computationally prohibitive even for small data sets. Gyllenberg et al. (1994b) therefore developed an algorithm which finds an approximation of the minimum value of SC for 
a given value $k$ of the number of classes. Repeating the algorithm for different values of $k$ and choosing the one giving the smallest SC one obtains a classification that should be close to the true SC-minimizing classification.

The algorithm consists of initialization and three steps of reestimation which are repeated as long as the classification is improving.

1. Initialization. $k$ binary feature vectors are chosen at random. They represent the hypothetical mean organisms (HMOs) of $k$ classes.

2. Identification. The strains in the data are identified, that is, each strain is associated to the nearest HMO. Here the distance is interpreted in terms of codelength, that is, the number of bits needed to represent a strain in relation to the HMO.

3. Parameter estimation. The parameters $\lambda_{j}$ and $\theta_{i j}$ of the classification obtained in step 2 are calculated.

4. Redefining the HMOs. The HMOs of the classification obtained in step 2 are calculated.

The procedure is repeated starting from step 2 using the HMOs found in step 4 at the previous iteration until the HMOs do not change.

The above algorithm was implemented in a computer program written in ANSI-C, almost 6500 lines of code. The compilers Borland $\mathrm{C}++4.51$ and GNU-CC 2.7.1 were used. Since the initialization (step 1) is random, the algorithm does not always yield the same result. For each value of $k$ the algorithm was therefore repeated at least 20 times and the least value of SC was considered as the best value for that particular $k$. The standard deviation of SC was typically about 0.15 and the classifications corresponding to SC values close to the least value all showed the same general structure.

\section{RESULTS}

\section{Minimization of SC}

Fig. 1 shows how the minimum of SC changed as the Enterobacteriaceae material (ENTE) was divided into an increasing number of classes. With two classes the minimum of $\mathrm{SC}$ was approximately 33 , but the minimum decreased rather steeply as the number of classes rose and attained its least value of 21.421 at 69 classes. We denote this SC-minimizing classification SCENTE. With further increase in the number of classes the minimum of SC increased, but slightly. ENTE included 104 traditional species (or corresponding biogroups) and the SC of this traditional classification (CFARM) was $23 \cdot 124$, compared with an SC of 21.888 when ENTE was classified into 104 classes by the method of SC minimization.

What do these SC values indicate? The difference $23.124-21.421=1.703$ in SC between SCENTE and CFARM means that 1.703 more bits per item are needed to encode the classification and the data using CFARM than using the SC-minimizing classification SCENTE (69 classes). In other words SCENTE is $2^{1 \cdot 703}=3 \cdot 27$

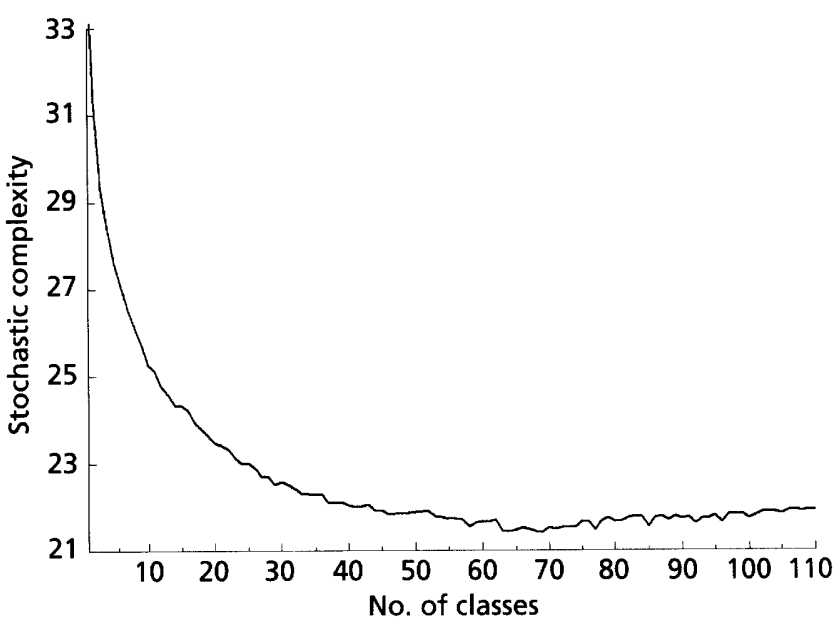

Fig. 1. The minimum of the $\mathrm{SC}$ of the material ENTE (Enterobacteriaceae) as a function of the number of classes.

times 'better' than CFARM. The SC classification into 104 classes, on the other hand, is 2.34 times 'better' (from an information theory point of view) than CFARM $\left(2^{23 \cdot 124-21 \cdot 888}=2 \cdot 339\right)$.

\section{SC classification into 104 classes}

Before turning to the SC-minimizing classification of ENTE its SC classification into 104 classes will be briefly compared with CFARM, which also contains 104 classes (Table 1). Although the SC classification confirms the general structure of CFARM, differences are obvious. Only 17 of the 104 species (or biogroups) of CFARM were clearly distinguishable in the SC classification. These were Cedecea davisae, Citrobacter diversus, C. amalonaticus 1, Enterobacter georgeviae, Escherichia fergusonii, E. hermanii, E. vulneris, Leclercia adecarboxylata, Moellerella wisconsensis, Morganella morganii, Serratia ficaria, S. fonticola, S. odorifera 1, S. odorifera 2, S. plymuthica, S. rubidae and Tatumella ptyseos. Other species were divided into two or more classes (particularly Escherichia coli, which produced some 20 classes). Certain genera were collected together without separation of species (e.g. Kluyvera and Edwardsiella) whereas other genera (e.g. Enterobacter, Klebsiella, Proteus, Providencia) were split into several classes but not according to the species division of CFARM.

\section{SC-minimizing classification of Enterobacteriaceae (SCENTE)}

The outcome of the SC-minimizing classification of ENTE, and a comparison with CFARM, are given in Table 2. It gives rise to the following conclusions. (1) Only 11 of the 104 species in CFARM are strictly confirmed (i.e. they formed their own classes, possibly 


\section{Table 1. The CFARM classification of ENTE}

The size of a class is the number of specimens in that class. The distortion denotes the items' average distance from the $\mathrm{HMO}$ measured in number of differing bits.

\begin{tabular}{|c|c|c|}
\hline Species & Size & Distortion \\
\hline Budvicia aquatica & 6 & $2 \cdot 83$ \\
\hline Buttiauxella agrestis & 5 & $1 \cdot 20$ \\
\hline Cedecea davisae & 31 & $2 \cdot 45$ \\
\hline Cedecea lapagei & 10 & $2 \cdot 70$ \\
\hline Cedecea neteri & 4 & $0 \cdot 75$ \\
\hline Cedecea species 3 & 1 & 0.00 \\
\hline Cedecea species 5 & 1 & $0 \cdot 00$ \\
\hline Citrobacter amalonaticus & 31 & $2 \cdot 48$ \\
\hline Citrobacter amalonaticus 1 & 40 & $2 \cdot 58$ \\
\hline Citrobacter diversus & 54 & 3.00 \\
\hline Citrobacter freundii & 187 & $4 \cdot 23$ \\
\hline Edwardsiella hoshinae & 9 & $1 \cdot 11$ \\
\hline Edwardsiella ictaluri & 13 & 1.46 \\
\hline Edwardsiella tarda & 35 & $0 \cdot 97$ \\
\hline Edwardsiella tarda 1 & 7 & $0 \cdot 14$ \\
\hline Enterobacter aerogenes & 26 & $1 \cdot 89$ \\
\hline Enterobacter amnigenus 1 & 14 & $1 \cdot 50$ \\
\hline Enterobacter amnigenus 2 & 6 & $1 \cdot 67$ \\
\hline Enterobacter asburiae & 45 & $2 \cdot 42$ \\
\hline Enterobacter cloacae & 156 & 3.75 \\
\hline Enterobacter dissolvens & 1 & 0.00 \\
\hline Enterobacter gergoviae & 72 & $0 \cdot 86$ \\
\hline Enterobacter intermedium & 10 & $0 \cdot 60$ \\
\hline Enterobacter nimipressuralis & 1 & $0 \cdot 00$ \\
\hline Enterobacter sakazakii & 90 & $2 \cdot 17$ \\
\hline Enterobacter taylorae & 98 & $1 \cdot 16$ \\
\hline Erwinia ananas & 5 & 3.00 \\
\hline Erwinia chrysanthemi & 2 & $2 \cdot 00$ \\
\hline Erwinia cypripedii & 1 & 0.00 \\
\hline Erwinia herbicola & 15 & $5 \cdot 67$ \\
\hline Erwinia milletiae & 1 & $0 \cdot 00$ \\
\hline Erwinia rhapontici & 1 & $0 \cdot 00$ \\
\hline Erwinia rubrifaciens & 1 & $0 \cdot 00$ \\
\hline Erwinia stewartii & 3 & $1 \cdot 00$ \\
\hline Erwinia uredovora & 2 & $3 \cdot 00$ \\
\hline Escherichia blattae & 3 & $1 \cdot 33$ \\
\hline Escherichia coli & 1708 & $7 \cdot 07$ \\
\hline Escherichia fergusonii & 40 & $3 \cdot 80$ \\
\hline Escherichia hermanii & 68 & $3 \cdot 62$ \\
\hline Escherichia vulneris & 77 & $3 \cdot 51$ \\
\hline Ewingella americana & 87 & $5 \cdot 35$ \\
\hline Hafnia alvei & 99 & $4 \cdot 89$ \\
\hline Hafnia alvei 1 & 1 & $0 \cdot 00$ \\
\hline Klebsiella oxytoca & 90 & $1 \cdot 00$ \\
\hline Klebsiella ozaenae & 53 & $5 \cdot 68$ \\
\hline Klebsiella planticola & 17 & 0.59 \\
\hline Klebsiella pneumoniae & 167 & $4 \cdot 01$ \\
\hline Klebseilla pneumoniae 1 & 1 & 0.00 \\
\hline Klebsiella rhinoscleromatis & 12 & $1 \cdot 58$ \\
\hline Klebsiella terrigena & 9 & $1 \cdot 56$ \\
\hline Kluyvera ascorbata & 119 & 1.77 \\
\hline
\end{tabular}

Table 1. (cont.)

\begin{tabular}{|c|c|c|}
\hline Species & Size & Distortion \\
\hline Kluyvera cryocrescens & 35 & $1 \cdot 86$ \\
\hline Kluyvera species 3 & 1 & $0 \cdot 00$ \\
\hline Koserella trabulsii & 12 & $1 \cdot 75$ \\
\hline Leclercia adecarboxylata & 26 & $2 \cdot 85$ \\
\hline Leminorella grimontii & 4 & $1 \cdot 75$ \\
\hline Moellerella wisconsensis & 17 & $2 \cdot 59$ \\
\hline Morganella morganii & 66 & $3 \cdot 23$ \\
\hline Morganella morganii 1 & 12 & $2 \cdot 33$ \\
\hline Obesumbacterium proteus 1 & 3 & $2 \cdot 33$ \\
\hline Obesumbacterium proteus 2 & 3 & $0 \cdot 67$ \\
\hline Proteus mirabilis & 41 & $4 \cdot 20$ \\
\hline Proteus myxofaciens & 1 & $0 \cdot 00$ \\
\hline Proteus penneri & 51 & $3 \cdot 14$ \\
\hline Proteus vulgaris & 29 & $2 \cdot 93$ \\
\hline Providencia alcalifaciens & 17 & $1 \cdot 71$ \\
\hline Providencia rettgeri & 28 & 5.79 \\
\hline Providencia rustigianii & 29 & $2 \cdot 90$ \\
\hline Providencia stuartii & 67 & $4 \cdot 34$ \\
\hline Rabnella aquatilis & 19 & $2 \cdot 95$ \\
\hline Salmonella arizonae & 2 & $6 \cdot 00$ \\
\hline Salmonella choleraesuis & 22 & $2 \cdot 96$ \\
\hline Salmonella enteritidis & 99 & $6 \cdot 56$ \\
\hline Salmonella pullorum & 1 & $0 \cdot 00$ \\
\hline Salmonella typhimurium & 8 & $2 \cdot 75$ \\
\hline Salmonella typhi & 13 & $2 \cdot 00$ \\
\hline Serratia ficaria & 15 & $2 \cdot 87$ \\
\hline Serratia fonticola & 33 & $3 \cdot 18$ \\
\hline Serratia grimesii & 8 & $4 \cdot 75$ \\
\hline Serratia liquefaciens & 6 & $2 \cdot 17$ \\
\hline Serratia marcescens & 123 & $4 \cdot 55$ \\
\hline Serratia marcescens 1 & 22 & $5 \cdot 60$ \\
\hline Serratia odorifera 1 & 20 & $2 \cdot 75$ \\
\hline Serratia odorifera 2 & 28 & $2 \cdot 86$ \\
\hline Serratia plymutbica & 16 & $4 \cdot 44$ \\
\hline Serratia proteamaculans 1 & 5 & $9 \cdot 80$ \\
\hline Serratia proteamaculans 2 & 5 & $4 \cdot 20$ \\
\hline Serratia rubidae & 12 & $3 \cdot 17$ \\
\hline Shigella boydii & 120 & $3 \cdot 63$ \\
\hline Shigella dysenteriae & 57 & $3 \cdot 67$ \\
\hline Shigella flexneri & 181 & $4 \cdot 56$ \\
\hline Shigella sonnei & 72 & $3 \cdot 88$ \\
\hline Shigella prov & 67 & $3 \cdot 22$ \\
\hline Tatumella ptyseos & 53 & 1.98 \\
\hline Xenorhabdus luminescens 5 & 5 & $2 \cdot 20$ \\
\hline Yersinia aldovae & 5 & $3 \cdot 80$ \\
\hline Yersinia enterocolitica & 207 & $4 \cdot 59$ \\
\hline Yersinia frederiksenii & 30 & $3 \cdot 43$ \\
\hline Yersinia intermedia & 35 & $4 \cdot 80$ \\
\hline Yersinia kristensenii & 25 & $4 \cdot 32$ \\
\hline Yersinia pestis & 2 & $1 \cdot 50$ \\
\hline Yersinia pseudotuberculosis & 24 & $1 \cdot 83$ \\
\hline Yersinia robdei & 2 & $1 \cdot 50$ \\
\hline Yersinia ruckeri & 4 & $2 \cdot 75$ \\
\hline
\end{tabular}


with the inclusion of a few odd items). (2) Escherichia coli is certainly also confirmed but it splits into eight pure (i.e. containing only E. coli strains) and four almost pure classes (containing in addition a few odd strains). Specimens labelled E. coli show affinity particularly to shigellas (but also salmonellas and yersinias). (3) Several of the genera, e.g. Enterobacter, Klebsiella, and especially Serratia, Salmonella, Shigella and Yersinia, are split into several pure and almost pure genus classes, which do not, however, always correspond to the species labels of the included specimens.

Adding or subtracting items to a classified collection may in principle alter the already established classification with respect to both its structure and the number of classes. A classification method of any use in practical taxonomy should of course be rather rigid with respect to adding new items. To test whether the SC-based classification method fulfils this requirement we performed two further classifications. One classification comprised all escherichias (including Leclercia), and all salmonellas, shigellas and yersinias in ENTE, 2910 strains altogether. This material is referred to as ESSY. The other classification comprised the $1708 \mathrm{E}$. coli labelled strains (material COLI).

\section{SC-minimizing classification of ESSY (SCESSY)}

At the SC minimum (20.273) ESSY is divided into 26 classes. The results of SCESSY are condensed into Table 3. The genera Escherichia, Salmonella, Shigella and Yersinia are briefly commented on below.

Escherichia. Four species are (almost) strictly confirmed: E. fergusonii, E. hermanii, E. vulneris and Leclercia adecarboxylata. Farmer et al. (1985) concluded that the last-mentioned species, although originally known as Escherichia adecarboxylata, does not belong to Escherichia. In all our runs L. adecarboxylata was strictly separated and attachment to other classes could not be found. E. coli produces six pure and five almost pure classes. These are discussed in more detail in connection with material COLI.

Salmonella. In SCENTE the salmonellas are divided among four classes $(34,40,55$ and 65$)$. Of these, class 34 corresponds exactly to SCESSY class 18 , as does class 40 to SCESSY class 17. The SCENTE classes 55 and 65 are combined in SCESSY classes 18 and 19. (By correspondence between a SCESSY class A and a SCENTE class $B$ we mean that every element of $A$ is also an element of B and that B does not contain other ESSY elements than those belonging to $A$.)

Shigella. In SCENTE shigellas occur in classes 2, 12, 14 and 23. These four classes have their identical counterparts in SCESSY classes 21, 10, 8 and 22, respectively.

Yersinia. For the yersinias as well there is a complete correspondence between SCENTE (classes 7, 77, 50 and 51 ) and SCESSY (classes 23, 24, 26 and 25, respectively).
Summarizing the above observations, we conclude that as far as the genera Salmonella, Shigella and Yersinia are concerned, the SC-minimizing classification of the material ESSY gives rise to a classification identical with that resulting from minimizing $\mathrm{SC}$ of the considerably larger material ENTE. This shows that the SC-based classification method has a certain robustness with respect to altering the material.

\section{SC-minimizing classification of Escherichia coli (SCCOLI)}

The SC minimum of the COLI material was $20 \cdot 219$ at 10 classes. The analysis of the 10 SCCOLI classes was performed by comparison of the distribution of the individual E. coli strains within each of the three optimal classifications (materials COLI, ESSY and ENTE, respectively). The results are presented in Table 4.

As can be seen from Table 4, there is an obvious correspondence between certain SCCOLI classes and classes of the SCESSY and SCENTE classifications. SCCOLI classes 1, 2, 3 and 4 are well concentrated (cf. also Table 5) and represent the bulk of E. colis $(932 / 1708)$, corresponding well to the centroid and HMO of E. coli as defined by Farmer et al. (1985). These SCCOLI classes are also quite homogeneous (distortion figures $<4 \cdot 2$ ). It is, therefore, easy to conclude that the 'real' $E$. coli is included within these SCCOLI classes.

Farmer et al. (1985) also considered an 'inactive' E. coli. The description given corresponds quite well to the SCCOLI class 6 (Table 5). This class (which contains $156 / 1708 \mathrm{E}$. colis) is also homogeneous (distortion figure: 3.763 ). The remaining E. coli-labelled specimens $(620 / 1708)$ are distributed over five SCCOLI classes (5, $7,8,9$ and 10). These classes are all heterogeneous (distortion figures $>4.8$ ). As shown in Table 5, the $E$. coli-labelled specimens related to shigellas are found particularly in the SCCOLI classes 5 and 8. E. colis related to Yersinia pseudotuberculosis are found in the SCCOLI classes 7 and 9. It thus seems that the heterogeneous SCCOLI classes 5, 7, 8, 9 and 10 contain E. colis which are related to other organisms (Shigella, Y. pseudotuberculosis, etc.) or are otherwise atypical specimens, perhaps also misidentified isolates.

\section{DISCUSSION}

The purpose of the present study is to point out the applicability of a particular mathematical method (minimization of stochastic complexity) to classification of bacteria. As a specific example we have chosen phenotypic classification of Enterobacteriaceae based on biochemical tests. The SC classification considers, as does every numerical taxonomy approach, all characters (tests) as equally valid contributors of information and it thus takes into account the whole binary vector 
Table 2. The SC-minimizing classification of ENTE

Taxonomy refers to the classification given in Table 1.

\begin{tabular}{|c|c|c|c|c|c|}
\hline Class & Size & Distortion & Taxonomy & $\begin{array}{c}\text { No. of } \\
\text { strains } \\
\text { in material }\end{array}$ & $\begin{array}{l}\text { No. of } \\
\text { strains } \\
\text { in class }\end{array}$ \\
\hline 1 & 264 & $3 \cdot 54$ & E. coli & 1708 & 264 \\
\hline 2 & 247 & $3 \cdot 80$ & $\begin{array}{l}\text { E. coli } \\
\text { S. boydii } \\
\text { S. dysenteriae } \\
\text { S. flexneri } \\
\text { S. prov } \\
\text { S. sonnei } \\
\text { Y. kristensenii }\end{array}$ & $\begin{array}{r}1708 \\
120 \\
57 \\
181 \\
67 \\
72 \\
25\end{array}$ & $\begin{array}{r}10 \\
104 \\
36 \\
37 \\
58 \\
1 \\
1\end{array}$ \\
\hline 3 & 243 & $2 \cdot 26$ & $\begin{array}{l}\text { E. aerogenes } \\
\text { K. oxytoca } \\
\text { K. planticola } \\
\text { K. pneumoniae } \\
\text { K. terrigena }\end{array}$ & $\begin{array}{r}26 \\
90 \\
17 \\
167 \\
9\end{array}$ & $\begin{array}{r}19 \\
89 \\
17 \\
110 \\
8\end{array}$ \\
\hline 4 & 228 & $4 \cdot 04$ & E. coli & 1708 & 228 \\
\hline 5 & 212 & $3 \cdot 37$ & $\begin{array}{l}\text { C. amalonaticus } \\
\text { E. coli } \\
\text { S. arizonae }\end{array}$ & $\begin{array}{r}31 \\
1708 \\
2\end{array}$ & $\begin{array}{r}1 \\
210 \\
1\end{array}$ \\
\hline 6 & 160 & 3.93 & $\begin{array}{l}\text { C. freundii } \\
\text { E. coli }\end{array}$ & $\begin{array}{r}187 \\
1708\end{array}$ & $\begin{array}{r}159 \\
1\end{array}$ \\
\hline 7 & 159 & $3 \cdot 53$ & $\begin{array}{l}\text { Y. enterocolitica } \\
\text { Y. frederiksenii } \\
\text { Y. intermedia } \\
\text { Y. kristensenii }\end{array}$ & $\begin{array}{r}207 \\
30 \\
35 \\
25\end{array}$ & $\begin{array}{r}112 \\
30 \\
2 \\
15\end{array}$ \\
\hline 8 & 156 & 1.93 & $\begin{array}{l}\text { B. agrestis } \\
\text { E. fergusonii } \\
\text { K. ascorbata } \\
\text { K. cryocrescens } \\
\text { K. species } 3\end{array}$ & $\begin{array}{r}5 \\
40 \\
119 \\
35 \\
1\end{array}$ & $\begin{array}{r}2 \\
1 \\
119 \\
33 \\
1\end{array}$ \\
\hline 9 & 148 & $3 \cdot 84$ & $\begin{array}{l}\text { C. lapagei } \\
\text { E. amnigenus } 1 \\
\text { E. cloacae } \\
\text { E. dissolvens } \\
\text { E. intermedium }\end{array}$ & $\begin{array}{r}10 \\
14 \\
156 \\
1 \\
10\end{array}$ & $\begin{array}{r}1 \\
10 \\
127 \\
1 \\
9\end{array}$ \\
\hline 10 & 146 & $2 \cdot 84$ & E. coli & 1708 & 146 \\
\hline 11 & 141 & $3 \cdot 72$ & $\begin{array}{l}\text { E. coli } \\
\text { S. flexneri } \\
\text { S. prov }\end{array}$ & $\begin{array}{r}1708 \\
181 \\
67\end{array}$ & $\begin{array}{r}139 \\
1 \\
1\end{array}$ \\
\hline 12 & 140 & $3 \cdot 17$ & $\begin{array}{l}\text { E. rubrifaciens } \\
\text { E. stewartii } \\
\text { E. coli } \\
\text { S. boydii } \\
\text { S. flexneri } \\
\text { S. prov } \\
\text { Y. rohdei }\end{array}$ & $\begin{array}{r}1 \\
3 \\
1708 \\
120 \\
181 \\
67 \\
2\end{array}$ & $\begin{array}{r}1 \\
3 \\
1 \\
2 \\
124 \\
8 \\
1\end{array}$ \\
\hline 13 & 138 & $5 \cdot 14$ & $\begin{array}{l}\text { E. coli } \\
\text { S. boydii } \\
\text { S. flexneri }\end{array}$ & $\begin{array}{r}1708 \\
120 \\
181\end{array}$ & $\begin{array}{r}133 \\
4 \\
1\end{array}$ \\
\hline 14 & 113 & $4 \cdot 80$ & $\begin{array}{l}\text { E. coli } \\
\text { S. boydii } \\
\text { S. dysenteriae } \\
\text { S. flexneri }\end{array}$ & $\begin{array}{r}1708 \\
120 \\
57 \\
181\end{array}$ & $\begin{array}{r}66 \\
9 \\
21 \\
17\end{array}$ \\
\hline 15 & 111 & $5 \cdot 69$ & $\begin{array}{l}\text { E. coli } \\
\text { S. sonnei }\end{array}$ & $\begin{array}{r}1708 \\
72\end{array}$ & $\begin{array}{r}109 \\
2\end{array}$ \\
\hline 16 & 109 & $4 \cdot 77$ & E. coli & 1708 & 109 \\
\hline
\end{tabular}


Table 2. (cont.)

\begin{tabular}{|c|c|c|c|c|c|}
\hline Class & Size & Distortion & Taxonomy & $\begin{array}{c}\text { No. of } \\
\text { strains } \\
\text { in material }\end{array}$ & $\begin{array}{l}\text { No. of } \\
\text { strains } \\
\text { in class }\end{array}$ \\
\hline 17 & 109 & $3 \cdot 69$ & $\begin{array}{l}\text { Y. aldovae } \\
\text { Y. entercolitica } \\
\text { Y. kristensenii } \\
\text { Y. rohdei }\end{array}$ & $\begin{array}{r}5 \\
207 \\
25 \\
2\end{array}$ & $\begin{array}{r}4 \\
95 \\
9 \\
1\end{array}$ \\
\hline 18 & 108 & 1.58 & $\begin{array}{l}\text { E. amnigenus } 2 \\
\text { E. cloacae } \\
\text { E. nimipressuralis } \\
\text { E. taylorae }\end{array}$ & $\begin{array}{r}6 \\
156 \\
1 \\
98\end{array}$ & $\begin{array}{r}6 \\
3 \\
1 \\
98\end{array}$ \\
\hline 19 & 98 & $3 \cdot 68$ & $\begin{array}{l}\text { S. marcescens } 1 \\
\text { S. marcescens }\end{array}$ & $\begin{array}{r}22 \\
123\end{array}$ & $\begin{array}{r}1 \\
97\end{array}$ \\
\hline 20 & 98 & $4 \cdot 85$ & $\begin{array}{l}\text { M. morganii } \\
P . \text { alcalifaciens } \\
P . \text { rustigianii } \\
P . \text { stuartii }\end{array}$ & $\begin{array}{r}66 \\
7 \\
29 \\
67\end{array}$ & $\begin{array}{r}1 \\
7 \\
29 \\
61\end{array}$ \\
\hline 21 & 95 & $5 \cdot 26$ & E. coli & 1708 & 95 \\
\hline 22 & 94 & $2 \cdot 28$ & $\begin{array}{l}\text { E. amnigenus } 1 \\
\text { E. sakazakii }\end{array}$ & $\begin{array}{l}14 \\
90\end{array}$ & $\begin{array}{r}4 \\
90\end{array}$ \\
\hline 23 & 90 & $4 \cdot 08$ & $\begin{array}{l}\text { E. coli } \\
\text { S. enteritidis } \\
\text { S. boydii } \\
\text { S. flexneri } \\
\text { S. sonnei }\end{array}$ & $\begin{array}{r}1708 \\
99 \\
120 \\
181 \\
72\end{array}$ & $\begin{array}{r}19 \\
1 \\
1 \\
1 \\
68\end{array}$ \\
\hline 24 & 89 & $4 \cdot 91$ & $\begin{array}{l}\text { H. alvei } \\
\text { K. pneumoniae }\end{array}$ & $\begin{array}{r}99 \\
167\end{array}$ & $\begin{array}{r}86 \\
3\end{array}$ \\
\hline 25 & 83 & $3 \cdot 84$ & $\begin{array}{l}\text { M. morganii } 1 \\
M . \text { morganii } \\
P . \text { rettgeri } \\
X . \text { luminescens } 5\end{array}$ & $\begin{array}{r}12 \\
66 \\
28 \\
5\end{array}$ & $\begin{array}{r}12 \\
65 \\
1 \\
5\end{array}$ \\
\hline 26 & 81 & $3 \cdot 68$ & $\begin{array}{l}\text { C. amalonaticus } 1 \\
\text { C. amalonaticus } \\
\text { C. freundii } \\
\text { E. coli }\end{array}$ & $\begin{array}{r}40 \\
31 \\
187 \\
1708\end{array}$ & $\begin{array}{r}39 \\
27 \\
11 \\
2\end{array}$ \\
\hline 27 & 78 & $3 \cdot 60$ & $\begin{array}{l}\text { P. penneri } \\
\text { P. vulgaris }\end{array}$ & $\begin{array}{l}51 \\
29\end{array}$ & $\begin{array}{l}49 \\
29\end{array}$ \\
\hline 28 & 77 & $3 \cdot 51$ & E. vulneris & 77 & 77 \\
\hline 29 & 72 & $0 \cdot 86$ & E. gergoviae & 72 & 72 \\
\hline 30 & 70 & $3 \cdot 36$ & $\begin{array}{l}\text { E. asburiae } \\
\text { E. cloacae }\end{array}$ & $\begin{array}{r}45 \\
156\end{array}$ & $\begin{array}{l}44 \\
26\end{array}$ \\
\hline 31 & 70 & $6 \cdot 00$ & $\begin{array}{l}\text { E. intermedium } \\
\text { E. rhapontici } \\
\text { K. oxytoca } \\
\text { K. ozaenae } \\
\text { K. pneumoniae } 1 \\
\text { K. pneumoniae } \\
\text { K. rhinoscleromatis } \\
\text { K. terrigena } \\
\text { K. cryocrescens }\end{array}$ & $\begin{array}{r}10 \\
1 \\
90 \\
53 \\
1 \\
167 \\
12 \\
9 \\
35\end{array}$ & $\begin{array}{r}1 \\
1 \\
1 \\
5 \\
1 \\
47 \\
12 \\
1 \\
1\end{array}$ \\
\hline 32 & 68 & $3 \cdot 61$ & E. hermanii & 68 & 68 \\
\hline 33 & 65 & $5 \cdot 95$ & E. coli & 1708 & 65 \\
\hline 34 & 64 & $3 \cdot 25$ & $\begin{array}{l}\text { C. freundii } \\
\text { S. enteritidis } \\
\text { S. typhimurium }\end{array}$ & $\begin{array}{r}187 \\
99 \\
8\end{array}$ & $\begin{array}{r}5 \\
51 \\
8\end{array}$ \\
\hline 35 & 57 & $3 \cdot 39$ & E. americana & 87 & 57 \\
\hline 36 & 55 & $3 \cdot 18$ & $\begin{array}{l}\text { C. amalonaticus } 1 \\
\text { E. ictaluri } \\
\text { E. tarda } \\
\text { E. coli } \\
\text { E. americana } \\
\text { H. alvei } 1 \\
\text { O. proteus } 2\end{array}$ & $\begin{array}{r}40 \\
13 \\
35 \\
1708 \\
87 \\
1 \\
3\end{array}$ & $\begin{array}{r}1 \\
13 \\
35 \\
1 \\
1 \\
1 \\
3\end{array}$ \\
\hline
\end{tabular}


Table 2. (cont.)

\begin{tabular}{|c|c|c|c|c|c|}
\hline Class & Size & Distortion & Taxonomy & $\begin{array}{c}\text { No. of } \\
\text { strains } \\
\text { in material }\end{array}$ & $\begin{array}{l}\text { No. of } \\
\text { strains } \\
\text { in class }\end{array}$ \\
\hline 37 & 55 & 3.07 & $\begin{array}{l}\text { C. diversus } \\
\text { E. fergusonii }\end{array}$ & $\begin{array}{l}54 \\
40\end{array}$ & $\begin{array}{r}54 \\
1\end{array}$ \\
\hline 38 & 54 & $5 \cdot 59$ & $\begin{array}{l}\text { K. ozaenae } \\
\text { K. pneumoniae }\end{array}$ & $\begin{array}{r}53 \\
167\end{array}$ & $\begin{array}{r}47 \\
7\end{array}$ \\
\hline 39 & 53 & 1.57 & $\begin{array}{l}\text { P. stuartii } \\
T . \text { ptyseos }\end{array}$ & $\begin{array}{l}67 \\
53\end{array}$ & $\begin{array}{r}1 \\
52\end{array}$ \\
\hline 40 & 52 & $5 \cdot 65$ & $\begin{array}{l}\text { E. coli } \\
\text { S. choleraesuis } \\
\text { S. enteritidis } \\
\text { S. typhi } \\
\text { Y. ruckeri }\end{array}$ & $\begin{array}{r}1708 \\
22 \\
99 \\
13 \\
4\end{array}$ & $\begin{array}{r}1 \\
22 \\
14 \\
13 \\
2\end{array}$ \\
\hline 41 & 50 & $4 \cdot 58$ & E. coli & 1708 & 50 \\
\hline 42 & 49 & $5 \cdot 40$ & E. coli & 1708 & 49 \\
\hline 43 & 47 & $5 \cdot 96$ & $\begin{array}{l}\text { S. marcescens } 1 \\
\text { S. marcescens } \\
\text { S. proteamaculans } 1\end{array}$ & $\begin{array}{r}22 \\
123 \\
5\end{array}$ & $\begin{array}{r}20 \\
26 \\
1\end{array}$ \\
\hline 44 & 46 & $3 \cdot 52$ & $\begin{array}{l}\text { C. davisae } \\
\text { C. lapagei } \\
\text { C. neteri } \\
\text { C. species } 3 \\
\text { C. species } 5\end{array}$ & $\begin{array}{r}31 \\
10 \\
4 \\
1 \\
1\end{array}$ & $\begin{array}{r}31 \\
9 \\
4 \\
1 \\
1\end{array}$ \\
\hline 45 & 44 & $4 \cdot 27$ & $\begin{array}{l}\text { P. mirabilis } \\
\text { P. myxofaciens } \\
\text { P. penneri }\end{array}$ & $\begin{array}{r}41 \\
1 \\
51\end{array}$ & $\begin{array}{r}41 \\
1 \\
2\end{array}$ \\
\hline 46 & 40 & $3 \cdot 30$ & $\begin{array}{l}\text { E. aerogenes } \\
\text { S. fonticola }\end{array}$ & $\begin{array}{l}26 \\
33\end{array}$ & $\begin{array}{r}7 \\
33\end{array}$ \\
\hline 47 & 39 & $5 \cdot 41$ & $\begin{array}{l}\text { S. arizonae } \\
\text { S. grimesii } \\
\text { S. liquefaciens } \\
\text { S. odorifera } 1 \\
\text { S. proteamaculans } 1 \\
\text { S. proteamaculans } 2\end{array}$ & $\begin{array}{r}2 \\
8 \\
6 \\
20 \\
5 \\
5\end{array}$ & $\begin{array}{r}1 \\
7 \\
6 \\
19 \\
1 \\
5\end{array}$ \\
\hline 48 & 39 & $3 \cdot 51$ & $\begin{array}{l}\text { E. coli } \\
\text { E. fergusonii }\end{array}$ & $\begin{array}{r}1708 \\
40\end{array}$ & $\begin{array}{r}1 \\
38\end{array}$ \\
\hline 49 & 38 & $4 \cdot 71$ & $\begin{array}{l}\text { S. odorifera } 1 \\
\text { S. odorifera } 2 \\
\text { S. rubidae }\end{array}$ & $\begin{array}{l}20 \\
28 \\
12\end{array}$ & $\begin{array}{r}1 \\
28 \\
9\end{array}$ \\
\hline 50 & 35 & $3 \cdot 20$ & $\begin{array}{l}\text { E. herbicola } \\
\text { E. coli } \\
\text { Y. pestis } \\
\text { Y. pseudotuberculosis }\end{array}$ & $\begin{array}{r}15 \\
1708 \\
2 \\
24\end{array}$ & $\begin{array}{r}1 \\
8 \\
2 \\
24\end{array}$ \\
\hline 51 & 35 & $4 \cdot 69$ & $\begin{array}{l}\text { C. amalonaticus } \\
\text { K. cryocrescens } \\
\text { Y. intermedia }\end{array}$ & $\begin{array}{l}31 \\
35 \\
35\end{array}$ & $\begin{array}{r}1 \\
1 \\
33\end{array}$ \\
\hline 52 & 35 & $5 \cdot 51$ & $\begin{array}{l}\text { S. ficaria } \\
\text { S. plymuthica } \\
\text { S. proteamaculans } 1 \\
\text { S. rubidae }\end{array}$ & $\begin{array}{r}15 \\
16 \\
5 \\
12\end{array}$ & $\begin{array}{r}15 \\
16 \\
1 \\
3\end{array}$ \\
\hline 53 & 30 & $4 \cdot 80$ & $\begin{array}{l}P . \text { rettgeri } \\
P . \text { stuartii }\end{array}$ & $\begin{array}{l}28 \\
67\end{array}$ & $\begin{array}{r}25 \\
5\end{array}$ \\
\hline 54 & 30 & $5 \cdot 17$ & $\begin{array}{l}\text { E. americana } \\
\text { S. marcescens } 1 \\
\text { S. proteamaculans } 1 \\
\text { Y. aldovae }\end{array}$ & $\begin{array}{r}87 \\
22 \\
5 \\
5\end{array}$ & $\begin{array}{r}27 \\
1 \\
1 \\
1\end{array}$ \\
\hline 55 & 28 & 3.04 & $\begin{array}{l}\text { E. blattae } \\
\text { E. coli } \\
\text { S. enteritidis } \\
\text { S. pullorum } \\
\text { S. sonnei }\end{array}$ & $\begin{array}{r}3 \\
1708 \\
99 \\
1 \\
72\end{array}$ & $\begin{array}{r}3 \\
1 \\
22 \\
1 \\
1\end{array}$ \\
\hline
\end{tabular}


Table 2. (cont.)

\begin{tabular}{|c|c|c|c|c|c|}
\hline Class & Size & Distortion & Taxonomy & $\begin{array}{c}\text { No. of } \\
\text { strains } \\
\text { in material }\end{array}$ & $\begin{array}{l}\text { No. of } \\
\text { strains } \\
\text { in class }\end{array}$ \\
\hline 56 & 26 & $2 \cdot 85$ & L. adecarboxylata & 26 & 26 \\
\hline 57 & 26 & $4 \cdot 34$ & $\begin{array}{l}\text { H. alvei } \\
\text { K. trabulsii } \\
\text { S. enteritidis }\end{array}$ & $\begin{array}{l}99 \\
12 \\
99\end{array}$ & $\begin{array}{r}13 \\
12 \\
1\end{array}$ \\
\hline 58 & 21 & $3 \cdot 43$ & $\begin{array}{l}\text { E. hoshinae } \\
\text { E. tarda } 1 \\
\text { O. proteus } 1 \\
\text { Y. ruckeri }\end{array}$ & $\begin{array}{l}9 \\
7 \\
3 \\
4\end{array}$ & $\begin{array}{l}9 \\
7 \\
3 \\
2\end{array}$ \\
\hline 59 & 19 & $3 \cdot 11$ & $\begin{array}{l}\text { M. wisconsensis } \\
P . \text { rettgeri }\end{array}$ & $\begin{array}{l}17 \\
28\end{array}$ & $\begin{array}{r}17 \\
2\end{array}$ \\
\hline 60 & 19 & $2 \cdot 84$ & $\begin{array}{l}\text { E. chysanthemi } \\
\text { R. aquatilis }\end{array}$ & $\begin{array}{r}2 \\
19\end{array}$ & $\begin{array}{r}1 \\
18\end{array}$ \\
\hline 61 & 15 & $3 \cdot 40$ & $\begin{array}{l}\text { B. agrestis } \\
\text { C. freundii }\end{array}$ & $\begin{array}{r}6 \\
187\end{array}$ & $\begin{array}{c}3 \\
12\end{array}$ \\
\hline 62 & 13 & 3.92 & $\begin{array}{l}\text { E. cypripedii } \\
\text { E. herbicola } \\
\text { E. milletiae }\end{array}$ & $\begin{array}{r}1 \\
15 \\
1\end{array}$ & $\begin{array}{r}1 \\
11 \\
1\end{array}$ \\
\hline 63 & 12 & $5 \cdot 25$ & $\begin{array}{l}\text { E. ananas } \\
\text { E. chrysanthemi } \\
\text { E. herbicola } \\
\text { E. uredovora } \\
\text { R. aquatilis }\end{array}$ & $\begin{array}{r}5 \\
2 \\
15 \\
2 \\
19\end{array}$ & $\begin{array}{l}5 \\
1 \\
3 \\
2 \\
1\end{array}$ \\
\hline 64 & 10 & $5 \cdot 20$ & $\begin{array}{l}\text { B. aquatica } \\
\text { L. grimontii }\end{array}$ & $\begin{array}{l}6 \\
4\end{array}$ & $\begin{array}{l}6 \\
4\end{array}$ \\
\hline 65 & 10 & $4 \cdot 30$ & S. enteritidis & 99 & 10 \\
\hline 66 & 3 & $5 \cdot 67$ & $\begin{array}{l}\text { E. asburiae } \\
\text { K. ozaenae } \\
\text { S. grimesii }\end{array}$ & $\begin{array}{r}45 \\
53 \\
8\end{array}$ & $\begin{array}{l}1 \\
1 \\
1\end{array}$ \\
\hline 67 & 2 & $5 \cdot 00$ & $\begin{array}{l}\text { E. americana } \\
\text { T. ptyseos }\end{array}$ & $\begin{array}{l}87 \\
53\end{array}$ & $\begin{array}{l}1 \\
1\end{array}$ \\
\hline 68 & 1 & 0.00 & E. americana & 87 & 1 \\
\hline 69 & 1 & 0.00 & S. proteamaculans 1 & 5 & 1 \\
\hline
\end{tabular}

Table 3. Distribution of strains over the 26 classes of the SC-minimizing classification of ESSY and the species in the classification given in Table 1

The entries are absolute numbers. Dist., distortion.

\begin{tabular}{|c|c|c|c|c|c|c|c|c|c|c|c|c|c|c|c|c|c|c|c|c|c|c|c|c|c|c|c|c|}
\hline Species & $\begin{array}{l}\text { ESSY } \\
\text { class }\end{array}$ & $\left\{\begin{array}{l}\text { No.... } \\
\text { Size... } \\
\text { Dist.... }\end{array}\right.$ & $\begin{array}{c}1 \\
265 \\
3.57\end{array}$ & $\begin{array}{c}2 \\
222 \\
4 \cdot 00\end{array}$ & $\begin{array}{c}3 \\
216 \\
3 \cdot 39\end{array}$ & $\begin{array}{c}4 \\
186 \\
3 \cdot 45\end{array}$ & $\begin{array}{c}5 \\
149 \\
5 \cdot 02\end{array}$ & $\begin{array}{c}6 \\
141 \\
3 \cdot 69\end{array}$ & $\begin{array}{c}7 \\
131 \\
5 \cdot 71\end{array}$ & $\begin{array}{c}8 \\
119 \\
4 \cdot 87\end{array}$ & $\begin{array}{c}9 \\
101 \\
4.63\end{array}$ & $\begin{array}{c}10 \\
82 \\
6 \cdot 05\end{array}$ & $\begin{array}{c}11 \\
53 \\
475\end{array}$ & $\begin{array}{c}12 \\
45 \\
5 \cdot 53\end{array}$ & $\begin{array}{c}13 \\
39 \\
3 \cdot 51\end{array}$ & $\begin{array}{c}14 \\
68 \\
3.62\end{array}$ & $\begin{array}{c}15 \\
78 \\
3 \cdot 55\end{array}$ & $\begin{array}{c}16 \\
26 \\
2 \cdot 85\end{array}$ & $\begin{array}{c}17 \\
59 \\
5 \cdot 93\end{array}$ & $\begin{array}{c}18 \\
71 \\
3.61\end{array}$ & $\begin{array}{c}19 \\
38 \\
4 \cdot 37\end{array}$ & $\begin{array}{c}20 \\
249 \\
3 \cdot 83\end{array}$ & $\begin{array}{c}21 \\
134 \\
2 \cdot 95\end{array}$ & $\begin{array}{c}22 \\
86 \\
4.09\end{array}$ & $\begin{array}{c}23 \\
159 \\
3 \cdot 52\end{array}$ & $\begin{array}{c}24 \\
106 \\
3 \cdot 56\end{array}$ & $\begin{array}{c}25 \\
33 \\
4 \cdot 55\end{array}$ & $\begin{array}{r}26 \\
53 \\
445\end{array}$ \\
\hline E. coli & & 1708 & 265 & 222 & 215 & 186 & 145 & 140 & 128 & 72 & 101 & 80 & 52 & 45 & . & . & . & . & 5 & . & 1 & 9 & . & 15 & . & . & . & 27 \\
\hline E. fergusoni & & 40 & . & $\cdot$ & . & . & · & $\cdot$ & $\cdot$ & . & $\cdot$ & . & . & . & 39 & . & 1 & . & . & . & . & $\cdot$ & . & . & . & . & . & . \\
\hline E. hermanii & & 68 & . & . & . & . & . & . & . & . & . & . & . & . & . & 68 & . & . & . & . & . & . & . & . & . & . & . & . \\
\hline E. vulneris & & 77 & . & . & . & . & . & . & . & . & . & . & . & . & . & . & 77 & $\cdot$ & . & . & . & . & . & . & . & . & . & . \\
\hline L. adecarboxylata & & 26 & . & . & . & . & . & . & . & . & . & . & . & . & . & . & . & 26 & . & . & . & . & . & . & . & . & . & . \\
\hline S. choleraesuis & & 22 & . & . & . & . & . & . & . & . & . & . & . & . & . & . & . & . & 22 & . & . & . & . & . & . & . & . & . \\
\hline S. enteritidis & & 99 & . & . & . & . & . & . & . & . & . & . & . & . & . & . & . & . & 14 & 52 & 32 & . & . & 1 & . & . & . & . \\
\hline S. typhimurium & & 8 & . & . & . & . & . & . & . & . & . & . & . & . & . & . & . & . & . & 8 & . & . & . & . & . & . & . & . \\
\hline S. typbi & & 13 & . & . & . & . & . & . & . & . & . & . & . & . & . & . & . & . & 13 & . & . & $\cdot$ & . & . & . & . & . & . \\
\hline S. boydii & & 120 & . & . & . & . & 4 & . & . & 9 & . & . & . & . & . & . & . & . & . & . & . & 104 & 2 & 1 & . & . & . & . \\
\hline S. dysenteriae & & 57 & . & . & . & . & . & . & . & 21 & . & . & . & . & . & . & . & . & . & . & . & 36 & . & . & . & . & . & . \\
\hline S. flexneri & & 181 & . & . & . & . & . & . & 1 & 17 & . & . & 1 & . & . & . & . & . & . & . & . & 37 & 124 & 1 & . & . & . & . \\
\hline S. prov & & 67 & . & . & . & . & . & 1 & . & . & . & . & . & . & . & . & . & . & . & . & . & 58 & 8 & . & . & . & . & . \\
\hline S. sonnei & & 72 & . & . & . & . & . & . & 2 & . & . & . & . & . & . & . & . & . & . & . & 1 & 1 & . & 68 & . & . & . & . \\
\hline Y.enterocolitica & & 207 & . & . & . & . & . & . & . & . & . & . & . & . & . & . & . & . & . & . & . & . & . & . & 112 & 95 & . & . \\
\hline Y. fredriksenii & & 30 & . & . & . & . & . & . & . & . & . & . & . & . & . & . & . & . & . & . & . & . & . & . & 30 & . & & . \\
\hline $\mathrm{Y}$. intermedia & & 35 & . & . & . & . & . & . & . & . & . & . & . & . & . & . & . & . & . & . & . & . & . & . & 2 & & 33 & . \\
\hline Y. kristensii & & 25 & . & . & . & . & . & . & . & . & . & . & . & . & , & . & . & . & . & . & . & 1 & . & . & 15 & 9 & . & . \\
\hline Y. pseudotuberculosis & & 24 & . & . & . & . & . & . & . & . & . & . & . & . & . & . & . & . & . & . & . & . & . & . & . & . & . & 24 \\
\hline Others & & 30 & . & . & 1 & . & . & . & . & . & . & 2 & . & . & . & . & . & . & 5 & 11 & 4 & 3 & . & . & . & 2 & . & 2 \\
\hline
\end{tabular}


Table 4. The distribution of $E$. coli-labelled strains over the classes of SCCOLI and the pure and almost pure $E$. coli classes of SCESSY and SCENTE

The entries are absolute numbers. Dist., distortion.

\begin{tabular}{|c|c|c|c|c|c|c|c|c|c|c|c|c|}
\hline SCCOLI & & $\begin{array}{c}\text { No..... } \\
\text { Size... } \\
\text { Dist.... }\end{array}$ & $\begin{array}{r}1 \\
259 \\
3 \cdot 52\end{array}$ & $\begin{array}{r}2 \\
240 \\
4 \cdot 19\end{array}$ & $\begin{array}{r}3 \\
233 \\
3.66\end{array}$ & $\begin{array}{r}4 \\
200 \\
3 \cdot 50\end{array}$ & $\begin{array}{r}5 \\
188 \\
6 \cdot 01\end{array}$ & $\begin{array}{r}6 \\
156 \\
3 \cdot 76\end{array}$ & $\begin{array}{r}7 \\
138 \\
5 \cdot 82\end{array}$ & $\begin{array}{r}8 \\
131 \\
6 \cdot 08\end{array}$ & $\begin{array}{r}9 \\
108 \\
4 \cdot 82\end{array}$ & $\begin{array}{r}10 \\
55 \\
5.95\end{array}$ \\
\hline \multicolumn{13}{|l|}{ SCESSY } \\
\hline No. & Size & Dist. & . & . & . & . & . & . & . & . & . & . \\
\hline 1 & 265 & $3 \cdot 57$ & 254 & 1 & 5 & . & . & . & 3 & . & 2 & . \\
\hline 2 & 222 & $4 \cdot 00$ & . & 220 & . & . & . & . & . & 2 & . & . \\
\hline 3 & 216 & $3 \cdot 39$ & . & . & 215 & . & . & . & . & . & . & . \\
\hline 4 & 186 & $3 \cdot 45$ & . & . & 2 & 181 & 1 & . & . & . & . & 2 \\
\hline 5 & 149 & $5 \cdot 02$ & 2 & 10 & . & . & 117 & 8 & 7 & 1 & . & . \\
\hline 6 & 141 & $3 \cdot 69$ & . & 1 & . & . & . & 133 & . & 5 & . & . \\
\hline 7 & 131 & $5 \cdot 71$ & 2 & 4 & 2 & . & 2 & . & 106 & 12 & . & . \\
\hline 11 & 53 & $4 \cdot 75$ & 1 & 4 & 2 & . & . & . & $\cdot$ & 43 & . & 3 \\
\hline 9 & 101 & $4 \cdot 63$ & . & . & . & . & . & . & . & . & 101 & . \\
\hline 12 & 45 & $5 \cdot 53$ & . & . & . & . & $\cdot$ & . & . & . & . & 45 \\
\hline 10 & 82 & 6.05 & . & . & 7 & 18 & 47 & . & 7 & . & . & 1 \\
\hline Others & & & . & . & . & 1 & 21 & 15 & 15 & 68 & 5 & 4 \\
\hline \multicolumn{13}{|l|}{ SCENTE } \\
\hline No. & Size & Dist. & & & & & & & & & & \\
\hline 1 & 264 & $3 \cdot 54$ & 248 & . & 4 & . & . & . & 12 & . & . & . \\
\hline 4 & 228 & $4 \cdot 04$ & . & 225 & · & 1 & 2 & . & . & . & . & . \\
\hline 5 & 212 & $3 \cdot 37$ & . & . & 210 & . & . & . & . & . & . & . \\
\hline 10 & 146 & $2 \cdot 84$ & . & . & 1 & 145 & . & . & . & . & . & . \\
\hline 13 & 138 & $5 \cdot 14$ & 3 & 1 & . & . & 71 & 13 & 37 & 3 & 4 & 1 \\
\hline 11 & 141 & $3 \cdot 72$ & . & 1 & . & . & . & 132 & . & 6 & . & . \\
\hline 15 & 111 & $5 \cdot 69$ & 6 & 8 & 6 & . & 1 & · & 73 & 15 & . & . \\
\hline 41 & 50 & $4 \cdot 58$ & 1 & 5 & 1 & . & . & . & . & 41 & . & 2 \\
\hline 16 & 109 & $4 \cdot 77$ & . & . & 2 & 3 & . & . & . & . & 104 & . \\
\hline 42 & 49 & $5 \cdot 40$ & . & . & . & 1 & 1 & . & . & . & . & 47 \\
\hline 33 & 65 & $5 \cdot 95$ & . & . & . & 2 & 58 & . & 2 & 2 & . & 1 \\
\hline 21 & 95 & $5 \cdot 26$ & . & . & 7 & 46 & 31 & 2 & 8 & 1 & . & . \\
\hline Others & . & . & 1 & . & 2 & 2 & 24 & 9 & 6 & 63 & . & 4 \\
\hline
\end{tabular}

representing the various strains; it does not consider divisions produced by individual attributes, however interesting they may be.

We emphasize that the SC-minimizing classification maximizes the information content of the classification and thus provides the 'best' classification in the sense of information theory, which does not imply that the classification would be the most relevant from the biological point of view.

The results of the present study have to be evaluated within the limits given by the bacterial material involved and the data by which this material is defined. As to the bacterial material, one may argue that it was skewed since almost a third of the strains in the study on Enterobacteriaceae were labelled Escherichia coli. On the other hand, as Farmer et al. (1985) emphasized, the proportion of $E$. coli is even higher in clinical practice. Taxonomy is not dependent on clinical practice, but clinical practice is dependent on a taxonomy, which is relevant with respect to the isolates it deals with, or as Staley \& Krieg (1984) put it: 'bacterial classifications are devised for microbiologists, not for the entities being classified'.

Another point worth discussion is the means to characterize Enterobacteriaceae. Over time new traits have been introduced and this trend is reflected in the steady increase in the number of genera and species of Enterobacteriaceae. This also concerns the test battery for phenetic description of Enterobacteriaceae which provided the data for the present study. However, no such change can be discerned during the period over which the material considered in this paper was collected. The material contained strains isolated during 20 years (1969-1988) and the strains of different years were distributed uniformly over the respective classes in all the classifications obtained by SC-minimization.

The structure of the SC-based classification of Enterobacteriaceae was very rigid with respect to altering the material and corresponded well to the general taxo- 
Table 5. Comparison of classes produced by CFARM, SCCOLI and SCESSY by their HMOs

The entries are the distance between the HMOs measured in number of differing bits.

\begin{tabular}{|ccccc|}
\hline \multirow{2}{*}{$\begin{array}{l}\text { SCCOLI } \\
\text { or }\end{array}$ SCESSY } & \multicolumn{4}{c}{ CFARM class: } \\
\cline { 2 - 5 } class & $\begin{array}{c}\text { Normal } \\
\text { E. coli }\end{array}$ & $\begin{array}{c}\text { Inactive } \\
\text { E. coli }\end{array}$ & $\begin{array}{c}\text { Shigella } \\
\text { A, B, C }\end{array}$ & $\begin{array}{c}\text { Shigella } \\
\text { sonnei }\end{array}$ \\
\hline SCCOLI/1 & 4 & 14 & $21 / 19$ & 16 \\
SCCOLI/2 & $1 / 3$ & 11 & 18 & 12 \\
SCCOLI/3 & $5 / 3$ & 15 & $22 / 20$ & 16 \\
SCCOLI/4 & $3 / 1$ & 13 & $19 / 17$ & 15 \\
SCCOLI/6 & $8 / 10$ & 2 & $8 / 10$ & 7 \\
SCCOLI/5 & $7 / 9$ & 3 & 10 & 5 \\
SCCOLI/7 & $8 / 10$ & 7 & 12 & 8 \\
SCCOLI/8 & $7 / 9$ & 4 & $9 / 11$ & 6 \\
SCCOLI/9 & $7 / 9$ & 11 & 18 & 11 \\
SCOLLI $/ 10$ & $7 / 9$ & 11 & 14 & 9 \\
SCESSY $/ 8$ & $12 / 14$ & 2 & $7 / 9$ & 7 \\
SCESSY $/ 10$ & $12 / 14$ & 5 & $6 / 8$ & 7 \\
SCESSY $/ 21$ & 15 & 8 & $5 / 3$ & 9 \\
SCESSY $/ 22$ & $8 / 10$ & 7 & 10 & 5 \\
\hline
\end{tabular}

nomic structure revealed by other classification methods. However, some differences were obvious. $E$. coli, the key species of Enterobacteriaceae to which all other members of the family have always been compared, was split into several entities by the SC-based classification. In contrast, other species of Escherichia (E. fergusonii, E. hermanii, E. vulneris) were distinctly separable and definable. In other entities of Enterobacteriaceae, within genera such as Proteus and Providencia, known species were combined into joint classes. SC classification is thus neither a splitter nor a lumper: it both splits and lumps.

The SC-based classification has been compared with present, generally accepted classifications of Enterobacteriaceae. This does not mean that the authors seek some kind of authorization for their method from agreement with the outcomes of other approaches. There are no 'official classifications' in microbiology. However, new approaches have to be checked against earlier thoughts. The SC classification applied to a set of name-labelled specimens compares taxospecies (or taxoclasses) with nomenspecies (cf. Sneath, 1984). This is the usual approach of numerical taxonomy. Sneath (1995b) concluded that results of numerical taxonomy have not necessarily confirmed earlier views. The present paper comes close to confirmation of earlier views, but may indicate some further problems requiring attention.

Vandamme et al. (1996) reviewed the various approaches to classification of bacteria, and illustratively concluded that a combination of the results from all of them (polyphasic taxonomy) is to be recommended. SC-based classification may provide a further tool for polyphasic taxonomy. It also provides a useful tool for comparing the information content of alternative classifications of common materials. The SC method is well suited for the treatment of large (and thus statistically representative) materials. Finally, as already emphasized in the Introduction, the SC-based classification sets no preconditions. There is no predefined level of similarity that an item has to fulfil in order to be included as a member of a class.

The amount of comparative molecular sequence data is increasing at an exponential rate and as a consequence the importance of phenotypic numerical taxonomy based on biochemical tests is declining. If the SC method were applicable only to classifying items characterized by their phenotype its value for bacterial taxonomy would be rather meagre. From a mathematical point of view minimizing SC is a method for classifying (binary) vectors (Gyllenberg et al., 1994b). Since the molecular sequence data of a bacterium can be given a binary code equivalent to the standard code using the four-letter alphabet ACGT, the SC approach can, at least in principle, be applied to molecular sequence data. It should, however, be noted that the statistical model (2) of the classification based on phenotypic data would most probably be a poor model for a classification based on genotypic data. It is an important and challenging task to construct a relevant model for classifications based on molecular sequence data. Once this has been done the SC method presented in this paper can immediately be applied.

\section{ACKNOWLEDGEMENTS}

The authors express their sincerest thanks to Dr J. J. Farmer III for the permission to use the data material on which this study was based.

\section{REFERENCES}

Balows, A., Truper, H. G., Dworkin, M., Harder, W. \& Schleifer, K.-H. (1992). The Prokaryotes, 2nd edn. New York: SpringerVerlag.

Brenner, D. J. (1992). Introduction to the family Enterobacteriaceae. In The Prokaryotes, 2nd edn, pp. 2673-2695. Edited by A. Balows, H. G. Trüper, M. Dworkin, W. Harder \& K.-H. Schleifer. New York: Springer-Verlag.

Dybowski, W. \& Franklin, D. A. (1968). Conditional probability and the identification of bacteria, J Gen Microbiol 54, 215-229.

Farmer, J. J., III, Davis, B. R., Hickman-Brenner, F. W. \& 12 other authors (1985). Biochemical identification of new species and biogroups of Enterobacteriaceae isolated from clinical specimens. J Clin Microbiol 21, 46-76.

Gower, J. C. (1974). Maximal predictive classification. Biometrics 30, 643-654.

Gyllenberg, M. \& Koski, T. (1996). Numerical taxonomy and the principle of maximum entropy. J Classif 13, 213-229.

Gyllenberg, M., Gyllenberg, H. G., Koski, T. \& Schindler, J. (1993). Non-uniqueness of numerical taxonomic structures. Binary 5, 138-144. 
Gyllenberg, M., Koski, T., Reilink, E. \& Verlaan, M. (1994a). Nonuniqueness in probabilistic numerical identification of bacteria. $J$ Appl Probab 31, 542-548.

Gyllenberg, M., Koski, T. \& Verlaan, M. (1994b). Classification of Binary Vectors by Stochastic Complexity. University of Turku Research Report A5 (ISBN 951-29-0358-X).

Krieg, N. R. \& Holt, J. G. (1984). Bergey's Manual of Systematic Bacteriology. Baltimore \& London: Williams \& Wilkins.

Lapage, S. P., Bascomb, S., Willcox, W. R. \& Curtis, M. A. (1973). Identification of bacteria by computer: general aspects and perspectives. J Gen Microbiol 77, 291-315.

Pankhurst, R. J. (1991). Practical Taxonomic Computing. Cambridge: Cambridge University Press.

Rissanen, J. (1989). Stochastic Complexity in Statistical Inquiry. Singapore: World Scientific.

Sneath, P. H. A. (1979). BASIC program for determining the best identification scores possible from the most typical examples when compared with an identification matrix of percent positive characteristics. Comput Geosci 6, 27-34.

Sneath, P. H. A. (1984). Numerical Taxonomy. In Bergey's Manual of Systematic Bacteriology vol. 1, pp. 5-7. Edited by
N. R. Krieg \& J. G. Holt. Baltimore \& London: Williams and Wilkins.

Sneath, P. H. A. (1995a). The history and future potential of numerical taxonomy: the contributions of H. G. Gyllenberg. Binary 7, 32-36.

Sneath, P. H. A. (1995b). Thirty years of numerical taxonomy. Syst Biol 44, 281-298.

Staley, J. T. \& Krieg, N. R. (1984). Classification of prokaryotic organisms: an overview. In Bergey's Manual of Systematic Bacteriology vol. 1, pp. 1-4. Edited by N. R. Krieg \& J. G. Holt. Baltimore \& London: Williams \& Wilkins.

Vandamme, P., Pot, B., Gillis, M., De Vos, P., Kersters, K. \& Swings, J. (1996). Polyphasic taxonomy, a consensus approach to bacterial systematics. Microbiol Rev 60, 407-438.

Willcox, W. R., Lapage, S. P. \& Holmes, B. (1980). A review of numerical methods in bacterial identification. Antonie Leeuwenhoek 46, 233-299.

Received 15 March 1996; revised 25 September 1996; accepted 21 October 1996. 